\title{
AOR
}

Selected Papers of \#AolR2019:

The $20^{\text {th }}$ Annual Conference of the Association of Internet Researchers Brisbane, Australia / 2-5 October 2019

\section{TRUSTED MACHINES? MACHINE LEARNING, MORE-THAN-HUMAN SPEED AND DEAD LABOR IN PLATFORM CAPITALISM}

Sy Taffel

Massey University

Decision making machines are today 'trusted' to perform or assist with a rapidly expanding array of tasks. Indeed, many contemporary industries could not now function without them. Nevertheless, this trust in and reliance upon digital automation is far from unproblematic. This paper combines insights drawn from qualitative research with creative industries professionals, with approaches derived from software studies and political economy to critically interrogate three ways that digital automation is currently employed and accompanying questions that relate to trust. Firstly, digital automation is examined as a way of saving time and/or reducing human labor, such as when programmers use automated build tools or graphical user interfaces. Secondly, automation enables new types of behavior by operating at more-than-human speeds, as exemplified by high-frequency trading algorithms. Finally, the mode of digital automation associated with machine learning attempts to both predict and influence human behaviors through recognizing patterns in computationally analyzable datasets, as epitomized by personalization algorithms within social media and search engines.

When exploring questions surrounding trust and digital automation, it is important to recognize the extent to which numerous contemporary industries are functionally dependent upon the labor-saving affordances of digital automation. For example, automatic focusing, metering, and flash compensation are routinely employed by photographers on location. When editing photos, image blending, stitching, perspective correction, and a range of presets, plug-ins, actions and looks are similarly employed to automate workflows to save time. When presenting their work online, automated tools are used to build websites, integrate them with corporate social media platforms and perform search engine optimization. These heavily automated tools allow photographers to work as agile, flexible, creative entrepreneurs. A homologous situation exists in game design, whereby game engines present a set of automated tools that Suggested Citation (APA): Taffel, S. (2019, October 2-5). Trusted Machines? Machine Learning, MoreThan-Human Speed and Dead Labor in Platform Capitalism. Paper presented at AolR 2019: The $20^{\text {th }}$ Annual Conference of the Association of Internet Researchers. Brisbane, Australia: AolR. Retrieved from http://spir.aoir.org. 
allow independent developers to produce games at a scale that would otherwise be impossible. These technologies are trusted to the point of being essential for these people's livelihoods. This is identified as a positive marker of identity by many of these creative workers, whilst also embodying a number of traits associated with neoliberalism (Mould 2018). These technologies are trusted as reliable ways of saving time and therefore money, but a critical examination of how these tools are located within a broader techno-cultural context of precarity, competition and commodification - what Bernard Stiegler (2017) describes as hyper-proletarianization - raises questions about the desirability of these dependencies. Arguably this continues the long history of automation displacing human action into what Karl Marx described as 'dead labor', productive activity which is amenable to social exploitation.

At the same time, trusting forms of automation associated with more-than-human speed can lead to spectacular failure. Emblematic of this are the high frequency trading (HFT) algorithms that today account for approximately half of all stock market trades. HFT systems execute tasks "that no human could ever hope to attempt" (Beddington et al. 2012); whereas it takes a human around 200 milliseconds to even perceive a change, HFT algorithms execute trades in just a few milliseconds. HFT systems execute huge volumes of trades and although each transaction produces miniscule profits, the massive number of minute quantities adds up to significant amounts, while greatly increasing the overall volume of financial exchanges (Toscano 2013). HFTs have contributed to the formation of 'flash crashes'; episodes where enormous sums have been wiped off the value of global stocks. Within these episodes HFT algorithms not only contribute to the crashes' speed, they also behave 'erratically', buying 'stub quotes' that are not intended to be purchased, and never would be by human traders. When decision-making machines deviate from desirable behaviors, they often do so in ways that differ from human errors. Furthermore, the speeds that HFTs operate at entail that real-time governance is impossible. Automating tasks can often mean a higher statistical success rate when compared to human labor, but humans are quite good at knowing where, when and why human errors are likely to occur. Conversely, automated systems make errors that appear inexplicable and yet can have enormously significant consequences, such as wiping hundreds of millions of dollars off the value of stocks in just a few minutes. These systems are 'trusted' insofar as they are relied upon, but they increase systemic instability and the likelihood of catastrophic 'black swan' events which raises serious questions as to whether this trust is misplaced.

One of the key questions surrounding the implementation of machine learning-based systems surrounds their lack of transparent decision-making. Unlike other digital systems, which follow rules that are explicitly written for the computational system to follow, machine learning systems and neural networks construct their own modes of categorization. Effectively, they learning to recognize patterns within training data they are given, and subsequently apply them to real-world data. As the process of fitting patterns is devised by the computational system itself, the underlying logic is often completely opaque to humans; we have no way of knowing exactly how the machine has arrived at a decision (Bridle 2018). While these nonhuman systems are more accurate than expert humans in many cases, increased accuracy does not equate to infallibility, and when machine learning algorithms underfit and overfit patterns based upon training data serious issues arise. Even when these systems function as designed, 
they often reproduce pre-existing social and cultural forms of discrimination but do so under the guise of technocratic objectivity, effectively preventing a critical examination of structural inequities whilst promoting homophily (Chun 2018). As Safiya Noble (2018:47) articulates, "automated decision-making systems are disproportionately harmful to the most vulnerable and the least powerful, who have little ability to intervene in them." Addressing these systemic problems requires more than just reprogramming particular algorithms, it entails addressing the neoliberal technocultural context that produces them.

While different forms of creative machines are increasingly trusted to underpin industries, culture and society, there are a range of reasons why we should query the desirability of increasing dependence on and trust in these technologies as they are currently employed. These for-profit, corporate-controlled tools performatively reproduce a neoliberal worldview. Discussing misplaced trust in digital automation frequently conjures an imagined binary opposition between humans and machines, however, this reductive fantasy conceals the far more concrete conflict between differing technocultural assemblages composed of humans and machines. Across the examples explored in this talk, what emerges are numerous ways in which creative machines are used to perpetuate social inequalities.

\section{References}

Beddington, J., Furse, C., Bond, P., Cliff, D., Goodhart, C., Houstoun, Zigrand, J.-P. (2012). Foresight: the future of computer trading in financial markets: final project report.

Bridle, J. (2018). New Dark Age: Technology and the end of the future. New York: Verso Books.

Chun, W. (2018) "Queering Homophily" in Apprich. C, Chun, W., Cramer, F., and Steyerl H. Pattern Discrimination: Minneapolis: University of Minnesota Press.

Mould, O. (2018). Against Creativity: New York: Verso Books.

Noble, S. U. (2018). Algorithms of Oppression: How search engines reinforce racism: New York: New York University Press

Stiegler, B. (2017). Automatic Society: The Future of Work: John Wiley \& Sons.

Toscano, A. (2013). Gaming the plumbing: High-frequency trading and the spaces of capital. Mute Magazine, 16 